OPEN ACCESS

Edited by:

Qinghua He,

Southwest University, China

Reviewed by:

Charles W. Mathias,

University of Texas Health Science Center at San Antonio, United States

Serge Brand,

University of Basel, Switzerland

*Correspondence:

Isabela M. M. Lima isabelammlima@gmail.com

Specialty section: This article was submitted to

Psychopathology, a section of the journal

Frontiers in Psychology

Received: 22 September 2017 Accepted: 11 December 2017

Published: 22 December 2017

Citation:

Lima IMM, Malloy-Diniz LF, de Miranda DM, Da Silva AG,

Neves FS and Johnson SL (2017) Integrative Understanding of Familial Impulsivity, Early Adversity and Suicide Risk.

Front. Psychol. 8:2240. doi: 10.3389/fpsyg.2017.02240

\section{Integrative Understanding of Familial Impulsivity, Early Adversity and Suicide Risk}

\author{
Isabela M. M. Lima ${ }^{1 *}$, Leandro F. Malloy-Diniz¹, Débora M. de Miranda1, \\ Antônio G. Da Silva², Fernando S. Neves ${ }^{3}$ and Sheri L. Johnson 4
}

${ }^{1}$ Graduate Program in Molecular Medicine, Federal University of Minas Gerais, Belo Horizonte, Brazil, ${ }^{2}$ Brazilian Association of Psychiatry, Rio de Janeiro, Brazil, ${ }^{3}$ Department of Mental Health, Faculty of Medicine, Federal University of Minas Gerais, Belo Horizonte, Brazil, ${ }^{4}$ Department of Psychology, University of California, Berkeley, Berkeley, CA, United States

Introduction: Impulsivity is a core characteristic of bipolar disorder and it was observed as elevated in individuals with the disorder and in their relatives. Both impulsivity and history of maltreatment are risk factors for suicide attempts, however, these two key variables may not be independent, given the fact that parental impulsivity and associated social context could increase the risk of child maltreatment. In this study it was examined the association between the impulsivity of relatives and child maltreatment taking into consideration the conjoint and unique effects of these two variables on the risk of suicide attempts among the patients.

Materials and Methods: Participants of the study consisted of 117 patients diagnosed with bipolar disorder and 25 first-degree relatives. Linear regression model was conducted to describe associations between facets of impulsivity of relatives and levels of child maltreatment reported by patients. The independent associations of suicide attempt history with the dimensions of impulsivity of the patient and maltreatment were tested by multinomial logistic regression.

Results: Impulsivity of relatives and, more specifically, inhibitory control can predict the maltreatment of the patient. Inhibitory control and emotional abuse were related, conjointly, to a greater likelihood of having a history of more than one suicide attempt.

Discussion: Considering that the impulsivity of relatives predicts child maltreatment, it is possible that a genetically shared impulsivity is an underlying feature associated with the history of multiple suicide attempts. These findings highlight the importance of considering child maltreatment, impulsivity and suicide attempt history in integrative models.

Keywords: bipolar, impulsive behavior, suicide, attempted, relatives, child maltreatment

\section{INTRODUCTION}

Bipolar bisorder (BD) consists of a heterogenic group of mood pathologies, which varies in terms of amount and severity of depressive and/or manic episodes, presence of psychotic symptoms, comorbid diagnostics, number of hospitalizations and suicide risk.

There are at least two main BD subtypes. Bipolar I disorder is characterized by the presence of at least one manic episode. Mania, in turn, is characterized by a period of at least 1 week of elevated, expansive or irritable mood, increased energy, and more talkativeness and engagement in risky activities (American Psychiatric Association [APA], 2013). Bipolar II 
is described as the presence of at least one previous major depressive episode for at least 2 weeks and one hypomanic episode that lasts a minimum of 4 days (American Psychiatric Association [APA], 2013).

Approximately one quarter of patients with Bipolar I report suicide attempts (Merikangas et al., 2011) and suicide accounts for 10 and $6.7 \%$ of deaths in Bipolar I and II, respectively (Angst et al., 2013). Hence, BD presents the highest absolute risk of suicide among different psychiatric conditions (Nordentoft et al., 2011). The focus of this paper is to understand the conjoint effects of two key factors for suicidality within BD: child maltreatment and impulsivity. With the aim to shed light into how impulsivity, maltreatment and suicidality interact, the goal of this study is to develop and test a more integrative model of these variables.

Impulsivity is considered to be a core feature of BD (Swann et al., 2003). To begin with, the diagnostic criteria for mania involves impulsivity. Even after manic episodes clear out, however, a growing body of research suggests that high levels of impulsivity persist (Swann et al., 2003). It is also noteworthy that impulsive behaviors can be transmitted through generations since it is observed to be elevated even in family members who do not meet diagnostic criteria for BD (Fortgang et al., 2016). According to the Self-Regulation Intergenerational Transmission Model, the diminished self-regulation that contributes to impulsive behavior is multifactorial, involving prenatal, social mechanisms of transmission, such as marital and parentchild interaction, as well as neurobiological mechanisms (Bridgett et al., 2015). Impulsivity is associated with low quality of life (Victor et al., 2011) and dysfunctional behaviors such as aggressiveness within BD (Johnson and Carver, 2016).

Particularly, impulsivity seems to be related to suicidality within BD (Johnson et al., 2016). Impulsive behavior triggered by emotion is related to history of suicidal ideation, self-harm, and suicide attempt in BD (Johnson et al., 2016) and also among those without BD (Kasen et al., 2011; Black and Mildred, 2013; Auerbach et al., 2016). These findings converge with the results of a longitudinal study, which indicates that impulsivity may predict suicide attempts over a 15 -year period in the general population (Kasen et al., 2011).

A separate literature indicates that early adversity and maltreatment are major concerns within BD. Rates of child abuse and neglect are quite high in those with the disorder, with exposure estimates as high as 51\% (Daruy-Filho et al., 2011). Early adversity and maltreatment have been associated with early bipolar onset and also to contribute to a poor prognosis within $\mathrm{BD}$, including more symptom severity, more relapse and lower levels of functioning (Daruy-Filho et al., 2011). Child maltreatment is also a predictor of the risk of suicide within $\mathrm{BD}$ (Norman et al., 2012). Indeed, a wide range of forms of child maltreatment, including sexual abuse, emotional abuse, physical abuse and emotional neglect, have been individually associated with the risk of attempted suicide within BD (Norman et al., 2012).

Although both impulsivity and childhood adversity seem to be important in understanding bipolar suicidality, these two variables have been studied separately in the literature on BD.
However, it is possible that impulsivity among family members may increase the risks of exposure to childhood adversity in offspring.

Considering this background, it was hypothesized that patients whose relatives have high levels of impulsivity may be in a social context that could increase the risk of abuse. First, the degree of overlap of early adversity and impulsivity among individuals with $\mathrm{BD}$ was assessed, then the conjoint and unique effects of early adversity and patient impulsivity as correlates of suicide attempt were considered. That is, it was examined: (1) whether the impulsivity of relatives can predict the levels of child maltreatment; (2) the extent to which the impulsivity of the patients and maltreatment dimensions are associated with suicide attempts.

\section{MATERIALS AND METHODS}

\section{Sample}

The sample consisted of a group of 117 bipolar patients and 25 first-degree relatives. The inclusion criteria taken were being aged between 18 and 60 years. Exclusion criteria were based on neurological conditions. The bipolar group consisted of individuals who had been previously diagnosed with BD by a psychiatrist and were being treated in a bipolar outpatient clinic which is part of Brazilian Public Health System. The bipolar diagnosis was confirmed through the administration of the MINI Plus 5.0 diagnostic interview (Sheehan et al., 1997). Each patient was asked to indicate a first-degree adult relative to compose the sample of relatives. However, only part of them attended to the scheduled appointment. Only a few of them met the inclusion criteria, resulting in a small sample of 25 relatives. Inclusion and exclusion criteria were the same applied to patients with the addition that they could not present psychiatric diagnosis, assessed by the administration of MINI Plus 5.0 diagnostic interview (Sheehan et al., 1997). First degree relative was considered as being parents' siblings or children of patients. No more than one relative per patient was assessed. Fifteen of the 25 relatives assessed were parents of patients, on the majority being their mothers. The Ethics Committee of the Federal University of Minas Gerais approved this study $\left(\mathrm{N}^{\circ}\right.$ 064/09) and all of the participants filled a written informed consent procedures before taking part in the study procedures.

\section{Measures}

Interviews and instruments were administered to patients in order to assess impulsivity, child maltreatment, suicide attempt history and mood states. Family members completed parallel measures of their personal levels of impulsivity and mood states.

\section{Barrat Impulsiveness Scale - BIS - 11 (Patton et al., 1995)}

The BIS - 11 is a 30 -item self-rated scale designed to assess impulsive thought and behavior. The Brazilian version of the scale was used, which has already been validated (MalloyDiniz et al., 2010, 2015). Previous analysis identified two factors from the extracted of the Brazilian version of the scale: 
Inhibition control $(\alpha=0.79)$, defined as the ability to inhibit a proponent behavior and attentional control, and Non-planning ( $\alpha=0.62$ ), defined as the decision making that requires costbenefit evaluation between short and long term consequences within an emotional context (Malloy-Diniz et al., 2015).

\section{Childhood Trauma Questionnaire - CTQ (Bernstein et al., 2003)}

The CTQ is a 28-item self-report questionnaire of frequency of traumas experienced before the age of 12, in 5-point likert scale, ranging from never to always. The Brazilian version of this scale was taken into consideration, which presents five subscales: emotional ( $\alpha=0.88)$, physical $(\alpha=0.92)$, and sexual abuse ( $\alpha=0.97)$, as well as emotional $(\alpha=0.94)$ and physical neglect ( $\alpha=0.66)$, (Grassi-Oliveira et al., 2006, 2014).

Suicide attempt history Suicide attempt history was assessed through a self-report question that classified patients as those who never attempted suicide, the ones that attempted once or those who attempted more than once. The question was "Have you ever attempted suicide?" The patient was asked to mark one of three options: Never attempted suicide, attempted once, attempted more than once. In the end, suicide attempt was defined as an act with clear intention to kill oneself.

\section{Beck Depression Inventory - BDI (Beck, 1961)}

The BDI is one of the most commonly used self-rated depression scales. The 21-item cover depressive symptoms such as energy level, hopelessness, suicide ideation and selfpunishment. Each item is rated on a scale of $0-3$ and higher scores reflect greater depression severity. The Brazilian version shows internal consistency of 0.81 (Gorenstein and Andrade, 1996).

\section{Young Mania Rating Scale - YMRS (Young et al., 1978)}

The YMRS scale is a semi-structured interview and rating system used to quantify severity of mania symptoms observed during clinical interview and in the past $48 \mathrm{~h}$. It consists of 11 questions that asses mood, activity level, psychomotor agitation, sexual interest, appearance, irritability, insight, sleep, aggressiveness and content of thoughts. Each item is scored on a scale of $0-4$, with the exception of items assessing irritability, speech, content of thoughts and disruptive behavior. These have double the scoring weight and are scored from 0 to 8 . Maximum score possible is 56 points. A trained clinician who had at least 2 years of experience in a bipolar outpatient clinic completed the interview and ratings. The Brazilian version of YMRS was used, which presented intraclass correlation coefficient of 0.80 and internal consistency of 0.67 (Vilela et al., 2005).

\section{Statistical Analysis}

All of the statistical analysis were performed in SPSS software, version 21.0. The following methods were selected to describe the associations of (1) impulsivity of relatives and levels of child maltreatment of patient; and (2) impulsivity and maltreatment dimensions of patient with history of suicide attempts and its reoccurrence. Since the sample size was unbalanced for relatives and patients, the analysis were divided in those two steps. First, principal components factor was conducted, using the oblimin rotation on the child maltreatment items of all 117 patients, in order to determine the number of statistically independent dimensions of trauma and reduce subsequent dependent variables in subsequent linear regression model. This was considered a preliminar requirement for step one, since the sample size was not enough to allow consideration of all dimensions of maltreatment as a dependent variable in further regression.

To examine the effect of impulsivity of relatives scores on child maltreatment of patients, a linear regression model was conducted with both BIS factors as independent variables. This step considered 25 patients and their respective relatives.

As a second step, a multinomial logistic regression model was computed to assess whether maltreatment and impulsivity predicted suicide attempt history among the 117 individuals with BD. The targeted categories were No history of suicide attempt; One previous suicide attempt; and More than one previous suicide attempt.

Depression and mania ratings were considered as potential confounds and were controlled as needed in both steps.

\section{RESULTS}

The demographic characteristics of the sample, as well as levels of impulsivity and child maltreatment, are described in Table $\mathbf{1 .}$ Approximately $67 \%$ of the patients met diagnostic criteria for Bipolar I and 32.3\% for Bipolar II disorder.

\section{Principal Components Analysis of the Childhood Abuse Scale}

Principal component analysis are shown in Table 2. The results showed no multicollinearity and the sample size was in accordance to Kaiser-Meyer-Olkin measure of sample adequacy $(\mathrm{KMO}=0.71)$, and $\mathrm{KMO}$ for individual variables were equal or higher than 0.67. Sphericity as tested by Bartlett's test indicated that the correlations between variables were adequate for Principal Components Analysis $\left(X^{2}=177.41, d f=10\right.$, $p<0.001)$. One factor surpassed Kaiser's criterion of Eigenvalue higher than 1 and explained $52.58 \%$ of the variance. Considering that the statistical requirements to consider maltreatment as an unique factor were met, maltreatment was taken as one factor on analysis regarding associations between impulsivity of relatives and maltreatment. These results show that the frequency of specific types of maltreatment are not dissociated from others. That is, those patients who tend to experience elevated levels of one type of maltreatment may also have higher probability to experience other forms of it.

\section{Impulsivity of Relatives Levels Correlate with Levels of Childhood Maltreatment of Patients}

Before conducting the analysis of the impulsivity effects, potential confounds of manic (YMRS) and depressive (BDI) symptoms 
were considered by testing correlations with maltreatment of patient scores. Neither the YMRS, $r(\mathrm{DF})=-0.07(24), p=0.74$ nor the BDI, $r(\mathrm{DF})=0.31(24), p=0.13$ were correlated with maltreatment. More specifically (as shown in Table 3), results of the linear regression show that first-degree BIS Inhibitory Control scores of relatives correlate significantly with patient report of General maltreatment, although Non-planning scores did not contribute to additional variance. Impulsivity of relatives explains a considerable proportion of the variance, $r^{2}=0.30$, $N=25, p<0.05$. Therefore, relatives impulsivity of relatives accounts for 30 percent of variance in self reported levels of maltreatment, showing that inhibitory control in relatives seem to impact on frequency of perceived maltreatment of patients.

TABLE 1 | Sample characteristics.

\begin{tabular}{|c|c|c|c|c|}
\hline & \multicolumn{2}{|c|}{ Patients $(N=117)$} & \multicolumn{2}{|c|}{ Relatives $(N=25)$} \\
\hline & $N$ & $\%$ & $N$ & $\%$ \\
\hline \multicolumn{5}{|l|}{ Level of education } \\
\hline $\begin{array}{l}\text { Secondary education or } \\
\text { below }\end{array}$ & 69 & 58.9 & 15 & 60.0 \\
\hline Partial undergraduat & 14 & 12.0 & 4 & 16 \\
\hline Undergraduate & 29 & 24.8 & 5 & 20.0 \\
\hline Graduate degree & 5 & 4.3 & 1 & 4 \\
\hline Gender - Women & 78 & 66.7 & 17 & 68.0 \\
\hline \multicolumn{5}{|l|}{ Suicide attempt history } \\
\hline No history of suicide attempt & 81 & 69.2 & - & - \\
\hline One previous suicide attempt & 20 & 17.1 & - & - \\
\hline \multirow{2}{*}{$\begin{array}{l}\text { More than one previous } \\
\text { suicide attempt }\end{array}$} & 16 & 12.7 & - & - \\
\hline & Mean & $S D$ & Mean & SD \\
\hline Age & 43.60 & 12.16 & 41.89 & 15.75 \\
\hline Beck Depression Inventory & 14.34 & 11.29 & 11.32 & 10.67 \\
\hline Young Mania Rating Scale & 1.90 & 3.18 & 3.09 & 3.86 \\
\hline BIS - Inhibition control & 40.55 & 9.14 & 35.52 & 6.96 \\
\hline BIS - Non-planning & 23.87 & 6.01 & 25.28 & 4.54 \\
\hline Physical neglect (3 items) & 4.60 & 1.96 & - & - \\
\hline Emotional neglect (7 items) & 13.87 & 6.12 & - & - \\
\hline Physical abuse (5 items) & 7.99 & 3.92 & - & - \\
\hline Emotional abuse (5 items) & 10.32 & 4.99 & - & - \\
\hline Sexual abuse (5 items) & 6.85 & 3.80 & - & - \\
\hline
\end{tabular}

TABLE 2 | Factor loading of Childhood Trauma Questionnaire subscales

\begin{tabular}{lc}
\hline Subscale & Factor 1: Maltreatment \\
\hline Emotional neglect & 0.877 \\
Physical neglect & 0.736 \\
Emotional abuse & 0.802 \\
Psysical abuse & 0.699 \\
Sexual abuse & 0.431
\end{tabular}

Extraction Method: Principal Component Analysis. Rotation Method: Oblimin with Kaiser Normalization.

\section{Maltreatment of Patients and Impulsivity Predict the Number of Previous Suicide Attempt}

As shown in Table 4, multinomial logistic regression was performed with BIS and Maltreatment scores as predictors of suicide attempt (never, once, more than once) as an outcome variable. Diminished inhibitory control was robustly correlated with number of suicide attempts, distinguishing specifically those who attempted suicide once or more from those who never attempted.

Child Maltreatment scores were considered separately to provide richer information regarding factors associated with presence and/or recurrence of suicide attempt. Only the Emotional abuse factor was significantly related to suicidality and it was only significant in differentiating repeated attempters from those with only one attempt. The correlations between impulsivity and maltreatment dimensions are shown in Table 5.

\section{DISCUSSION}

Many studies indicate that impulsivity and early adversity experiences are both correlated with suicidality within BD. Despite evidence that familial impulsivity may feature a context prone to greater risk of abuse, researchers have tended to consider these two variables separately. The goal of this study was to develop a more integrative understanding of familial impulsivity and early adversity, and also to consider whether patient's impulsivity and abuse contributed independently to the risk of suicide attempts for individuals with $\mathrm{BD}$. Since those that repeatedly attempted suicide may represent a subset of more severe patients, beyond discrimination of the presence of suicide attempt history, it was also an aim to describe aspects that could account for reincidence of suicide attempt.

Our findings confirmed that problems of patients with inhibitory control were tied to suicidality. Furthermore, findings extended previous work outside the BD diagnosis by showing that poor inhibitory control in relatives was related to the frequency of child maltreatment experienced by patients. Although these findings do not test direct influences of impulsivity of relatives and risk of suicide attempt in patients, it highlights that emotional abuse is related to a greater risk of recurrent suicide attempts even after accounting for deficits in inhibitory control in patients. These novel findings provide initial data to foster investigations on further unified and transgenerational perspective on impulsivity, adversity and suicidality.

TABLE 3 | Linear regression of Family Impulsivity Scores as Predictors of Maltreatment of Patients Scores $(N=25)$.

\begin{tabular}{lrrr}
\hline & B & SE B & b \\
\hline Constant & -0.10 & 1.12 & \\
BIS - Inhibitory Control & 0.05 & 0.02 & $0.43^{*}$ \\
BIS - Non-Planning & -0.06 & 0.03 & -0.36 \\
\hline
\end{tabular}

$r^{2}=0.30, N=25,{ }^{*} p<0.05$. 
This study, such as previous ones, suggests that the effects of impulsivity on suicidality within BD do not seem to generalize across measures of impulsivity, which indicates that it must be considered a multifaceted construct (Malloy-Diniz et al., 2009, 2011; Watkins and Meyer, 2013; Johnson et al., 2016). Previous works have found that impulsivity in the context of emotion is particularly predictive of suicidality, moreover this form of impulsivity would be helpful to be considered in future models of links between early adversity, impulsivity and bipolar suicidality.

The findings of this study dovetail with other researches that increasingly documents the complex interplay between maltreatment and impulsivity. Child maltreatment may not be independent of impulsivity of relatives. However, the results do not show the underlying causes that link them. Within $\mathrm{BD}$, relatively few relatives meet full diagnostic criteria for the disorder, but many experience high levels of impulsivity when compared to healthy controls (H1diroğlu et al., 2013). One

TABLE 4 | Multinomial logistic regression for history of suicide attempt.

\begin{tabular}{|c|c|c|c|c|}
\hline & \multirow[b]{2}{*}{ B (SE) } & \multicolumn{3}{|c|}{$95 \% \mathrm{Cl}$ for Odds Ratio } \\
\hline & & Lower & Odds Ratio & Upper \\
\hline \multicolumn{5}{|c|}{ No history of SA vs. more than one SA } \\
\hline Intercept & $7.56(2.81)$ & & & \\
\hline BIS - Inhibition control & $-0.11(0.04)^{*}$ & 0.83 & 0.89 & 0.97 \\
\hline BIS - Non-planning & $0.01(0.06)$ & 0.90 & 1.01 & 1.13 \\
\hline Physical neglect & $0.21(0.23)$ & 0.79 & 1.23 & 1.93 \\
\hline Emotional neglect & $-0.14(0.08)$ & 0.74 & 0.87 & 1.02 \\
\hline Sexual abuse & $-0.07(0.08)$ & 0.80 & 0.93 & 1.09 \\
\hline Physical abuse & $-0.14(0.09)$ & 0.73 & 0.87 & 1.04 \\
\hline Emotional abuse & $0.14(0.09)$ & 0.96 & 1.15 & 1.38 \\
\hline \multicolumn{5}{|c|}{ One previous SA vs. more than one SA } \\
\hline Intercept & $6.71(3.34)$ & & & \\
\hline BIS - Inhibition control & $-0.15(0.05)^{*}$ & 0.78 & 0.86 & 0.95 \\
\hline BIS - Non-planning & $-0.09(0.08)$ & 0.79 & 0.92 & 1.07 \\
\hline Physical neglect & $0.23(0.25)$ & 0.77 & 1.26 & 2.08 \\
\hline Emotional neglect & $-0.08(0.09)$ & 0.77 & 0.92 & 1.11 \\
\hline Sexual abuse & $0.02(0.08)$ & 0.87 & 1.02 & 1.20 \\
\hline Physical abuse & $-0.11(0.11)$ & 0.73 & 0.89 & 1.10 \\
\hline Emotional abuse & $0.27(0.11)^{*}$ & 1.06 & 1.31 & 1.62 \\
\hline
\end{tabular}

$R^{2}=0.29$ (Cox and Snell), 0.36 (Nagelkerke). Model $x^{2}=39.99, p<0.001 . d f=14$, $* p<0.05$.

TABLE 5 | Spearman's rho correlations between BIS-11 and CTQ patient's subscale scores.

\begin{tabular}{lcccccc}
\hline & $\mathbf{1}$ & $\mathbf{2}$ & $\mathbf{3}$ & $\mathbf{4}$ & $\mathbf{5}$ & $\mathbf{6}$ \\
\hline (1) BIS - Inhibition control & - & - & - & - & - & - \\
(2) BIS - Non-planning & $-0.35^{* *}$ & - & - & - & - & - \\
(3) Physical neglect & 0.10 & -0.14 & - & - & - & - \\
(4) Emotional neglect & 0.15 & $-0.21^{*}$ & $0.64^{* *}$ & - & - & - \\
(5) Sexual abuse & 0.18 & -0.02 & 0.18 & $0.20^{*}$ & - & - \\
(6) Physical Abuse & 0.13 & -0.00 & $0.36^{* *}$ & $0.45^{* *}$ & $0.23^{*}$ & - \\
(7) Emotional Abuse & $0.36^{* *}$ & -0.09 & $0.30^{* *}$ & $0.58^{* *}$ & $0.40^{* *}$ & $0.51^{* *}$ \\
\hline
\end{tabular}

${ }^{*} p<0.05 ; * * p<0.001$. possibility is that lack of inhibitory control in relatives is a marker of familial contexts which are prone to maltreatment behaviors. Higher familial impulsivity may lead to a more chaotic family environment that allows these other adversities to unfold. History of maltreatment, in turn, influence cognitive and emotional development, which intensify the risks of impulsivity and poor constraint over harmful behaviors (Braquehais et al., 2010; Daray et al., 2016). Inhibitory control shared by patients and relatives as an impulsivity dimension fosters dysfunctional behaviors such as maltreatment and suicide behavior. The reasons for relatives and patients to present dysfunctional may be diverse, possibly involving learnt behavior, developmental interferences of one behavior over another as well as shared genetic aspects that this study could not assess. That is, above and beyond the genetic influences, maltreatment has been shown to predict impulsivity over time (Braquehais et al., 2010; Daray et al., 2016).

Current findings indicated that inhibitory control and emotional abuse set the stage for recurrent suicide attempts rather than single attempts. The number of suicide attempts is a particularly robust predictor of suicide completion (Watkins and Meyer, 2013). Those that attempted suicide repeatedly also tend to present more severe dysfunctionality, as evidenced by their higher numbers of hospitalizations. Therefore, the clarification of inhibitory control as an specific aspect related to this subset of patients may be a promising target for treatments that aim to reduce recurrent suicide behavior and its dysfunctional consequences. If replicated, the current findings highlight the importance of considering specific types of early abuse as serious markers of more pronounced suicide risk.

Despite the potential clinical importance of these findings, the study is limited by the reliance on self-report measures of impulsivity and maltreatment, and also by the small number of relatives assessed. It is also important to consider that siblings and children were included in sample of relatives. Therefore, since it is less plausible that their impulsivity leads to maltreatment, it is not possible to conclude that the impulsivity observed in relatives leads to maltreatment. Instead, it may be more plausible to consider that impulsivity observed in relatives can be considered a family vulnerability marker to impulsive behaviors such as maltreatment. The small sample size precluded the ability to examine more refined dimensions of suicide attempts, such as lethality and age of onset. Furthermore, medication or comorbidities were not controlled. Despite the limitations, this study supports the relevance to develop approaches encompassing the transgenerational perspective on impulsivity and maltreatment in relation to suicide attempts in BD.

Current findings, if replicated, would have important clinical implications. First, emotional abuse and inhibitory control were independently associated with number of suicide attempt so they both represent important potential treatment targets. Second, interventions to address impulsivity may need to consider that this personality trait evolved in a family context. One possible approach for those who are seeking family therapy might be to integrate a consideration of family levels of impulsivity into that work. Impulsive behaviors of patients and family members 
could be addressed by teaching self-regulatory strategies, but also by considering communication effects and strategies in contexts in which multiple members of a family may have these concerns. By doing so, it may reduce the risk of high levels of conflict and potentially provide some protection from emotional abuse. As a whole, it is expected that these preliminary findings provide insight into some important links between variables that have been studied separately in understanding suicidality within bipolar disorder. Future studies should investigate causal relationships using larger samples and longitudinal designs.

\section{ETHICS STATEMENT}

This study was carried out in accordance with the recommendations of the Ethics Committee of the Federal University of Minas Gerais with written informed consent from all subjects. All subjects gave written informed consent in accordance with the Declaration of Helsinki. The protocol was

\section{REFERENCES}

American Psychiatric Association [APA] (2013). Diagnostic and Statistical Manual of Mental Disorders (DSM-5). Lake St. Louis, MO: American Psychiatric Pub.

Angst, J., Hengartner, M. P., Gamma, A., von Zerssen, D., and Angst, F. (2013). Mortality of 403 patients with mood disorders 48 to 52 years after their psychiatric hospitalisation. Eur. Arch. Psychiatry Clin. Neurosci. 263, 425-434.

Auerbach, R. P., Stewart, J. G., and Johnson, S. L. (2016). Impulsivity and suicidality in adolescent inpatients. J. Abnorm. Child Psychol. 45, 91-103. doi: 10.1007/ s10802-016-0146-8

Beck, A. T. (1961). An inventory for measuring depression. Arch. Gen. Psychiatry 4, 561-571. doi: 10.1001/archpsyc.1961.01710120031004

Bernstein, D. P., Stein, J. A., Newcomb, M. D., Walker, E., Pogge, D., Ahluvalia, T., et al. (2003). Development and validation of a brief screening version of the childhood trauma questionnaire. Child Abuse Negl. 27, 169-190. doi: 10.1016/ S0145-2134(02)00541-0

Black, E. B., and Mildred, H. (2013). Predicting impulsive self-injurious behavior in a sample of adult women. J. Nerv. Ment. Dis. 201, 72-75. doi: 10.1097/NMD. 0b013e31827ab1da

Braquehais, M. D., Oquendo, M. A., Baca-García, E., and Sher, L. (2010). Is impulsivity a link between childhood abuse and suicide? Compr. Psychiatry 51, 121-129. doi: 10.1016/j.comppsych.2009.05.003

Bridgett, D. J., Burt, N. M., Edwards, E. S., and Deater-Deckard, K. (2015). Intergenerational transmission of self-regulation: a multidisciplinary review and integrative conceptual framework. Psychol. Bull. 141, 602-654. doi: $10.1037 / \mathrm{a} 0038662$

Daray, F. M., Rojas, S. M., Bridges, A. J., Badour, C. L., Grendas, L., Rodante, D., et al. (2016). The independent effects of child sexual abuse and impulsivity on lifetime suicide attempts among female patients. Child Abuse Negl. 58, 91-98. doi: 10.1016/j.chiabu.2016.06.011

Daruy-Filho, L., Brietzke, E., Lafer, B., and Grassi-Oliveira, R. (2011). Childhood maltreatment and clinical outcomes of bipolar disorder. Acta Psychiatr. Scand. 124, 427-434. doi: 10.1111/j.1600-0447.2011.01756.x

Fortgang, R. G., Hultman, C. M., van Erp, T. G. M., and Cannon, T. D. (2016). Multidimensional assessment of impulsivity in schizophrenia, bipolar disorder, and major depressive disorder: testing for shared endophenotypes. Psychol. Med. 46, 1497-1507. doi: 10.1017/S0033291716000131

Gorenstein, C., and Andrade, L. (1996). Validation of a Portuguese version of the beck depression inventory and the state-trait anxiety inventory in Brazilian subjects. Braz. J. Med. Biol. Res. 29, 453-457.

Grassi-Oliveira, R., Cogo-Moreira, H., Salum, G. A., Brietzke, E., Viola, T. W., Manfro, G. G., et al. (2014). Childhood trauma questionnaire (CTQ) in approved by the Ethics Committee of the Federal University of Minas Gerais.

\section{AUTHOR CONTRIBUTIONS}

IL: Contributed on writing, performing statistics and delineating method. LM-D: Contributed on discussion, data collection and writing. DdM: Contributed on writing, discussion and data collection. ADS: Contributed on writing and reviewing. FN: Contributed on data collection, method and on delineating the study designed. SJ: Contributed on delineating, methods, statistics, discussion and writing.

\section{FUNDING}

This work was supported by CAPES - Proc. no. 6457302/15-0 and ABIPD - Associação Brasileira de Impulsividade e Patologia Dual.

Brazilian samples of different age groups: findings from confirmatory factor analysis. PLOS ONE 9:e87118. doi: 10.1371/journal.pone.0087118

Grassi-Oliveira, R., Stein, L. M., and Pezzi, J. C. (2006). Translation and content validation of the childhood trauma questionnaire into portuguese language. Rev. Sauide Puiblica 40, 249-255. doi: 10.1590/S0034-891020060002 00010

Hıdıroğlu, C., Demirci Esen, Ö., Tunca, Z., Neslihan Gûrz Yalçìn, S., Lombardo, L., Glahn, D. C., et al. (2013). Can risk-taking be an endophenotype for bipolar disorder? A study on patients with bipolar disorder type I and their first-degree relatives. J. Int. Neuropsychol. Soc. 19, 474-482. doi: 10.1017/ S1355617713000015

Johnson, S. L., and Carver, C. S. (2016). Emotion-relevant impulsivity predicts sustained anger and aggression after remission in bipolar I disorder. J. Affect. Disord. 189, 169-175. doi: 10.1016/j.jad.2015.07.050

Johnson, S. L., Carver, C. S., and Tharp, J. A. (2016). Suicidality in bipolar disorder: the role of emotion-triggered impulsivity. Suicide Life Threat. Behav. 47, 177-192. doi: 10.1111/sltb.12274

Kasen, S., Cohen, P., and Chen, H. (2011). Developmental course of impulsivity and capability from age 10 to age 25 as related to trajectory of suicide attempt in a community cohort. Suicide Life Threat. Behav. 41, 180-192. doi: 10.1111/j. 1943-278X.2011.00017.x

Malloy-Diniz, L. F., De Paula, J. J., Vasconcelos, A. G., De Almondes, K. M., Pessoa, R., Faria, L., et al. (2015). Normative data of the barratt impulsiveness scale 11 (BIS-11) for Brazilian adults. Rev. Bras. Psiquiatr. 37, 245-248. doi: 10.1590/1516-4446-2014-1599

Malloy-Diniz, L. F., Mattos, P., Leite, W. B., Abreu, N., Coutinho, G., Jardim De Paula, J., et al. (2010). Tradução e adaptação cultural da barratt impulsiveness scale (BIS-11) para aplicação em adultos brasileiros translation and cultural adaptation of barratt impulsiveness scale (BIS-11) for administration in Brazilian adults. J. Brasi. Psiquiat. 59, 99-105. doi: 10.1590/ S0047-20852010000200004

Malloy-Diniz, L. F., Neves, F. S., Abrantes, S. S. C., Fuentes, D., and Corrêa, H. (2009). Suicide behavior and neuropsychological assessment of type I bipolar patients. J. Affect. Disord. 112, 231-236. doi: 10.1016/j.jad.2008.03.019

Malloy-Diniz, L. F., Neves, F. S., De Moraes, P. H. P., De Marco, L. A., RomanoSilva, M. A., Krebs, M. O., et al. (2011). The 5-HTTLPR polymorphism, impulsivity and suicide behavior in euthymic bipolar patients. J. Affect. Disord. 133, 221-226. doi: 10.1016/j.jad.2011.03.051

Merikangas, K. R., Jin, R., He, J., Kessler, R. C., Lee, S., Sampson, N. A., et al. (2011). Prevalence and correlates of bipolar spectrum disorder in the world mental health survey initiative. Arch. Gen. Psychiatry 68, 241-251. doi: 10.1001/ archgenpsychiatry.2011.12 
Nordentoft, M., Mortensen, P. B., and Pedersen, C. B. (2011). Absolute risk of suicide after first hospital contact in mental disorder. Arch. Gen. Psychiatry 68, 1058-1064. doi: 10.1001/archgenpsychiatry.2011.113

Norman, R. E., Byambaa, M., De, R., Butchart, A., Scott, J., and Vos, T. (2012). The long-term health consequences of child physical abuse, emotional abuse, and neglect: a systematic review and meta-analysis. PLOS Med. 9:e1001349. doi: 10.1371/journal.pmed.1001349

Patton, J. H., Stanford, M. S., and Barratt, E. S. (1995). Factor structure of the barratt impulsiveness scale. J. Clin. Psychol. 51, 768-774. doi: 10.1002/10974679(199511)51:6<768::AID-JCLP2270510607>3.0.CO;2-1

Sheehan, D. V., Lecrubier, Y., Sheehan, K. H., Janavs, J., Weiller, E., Keskiner, A., et al. (1997). The validity of the mini international neuropsychiatric interview (MINI) according to the SCID-P and its reliability. Eur. Psychiatry 12, 232-241. doi: 10.1016/S0924-9338(97)83297-X

Swann, A. C., Pazzaglia, P., Nicholls, A., Dougherty, D. M., and Moeller, F. G. (2003). Impulsivity and phase of illness in bipolar disorder. J. Affect. Disord. 73, 105-111. doi: 10.1016/S0165-0327(02)00328-2

Victor, S. E., Johnson, S. L., and Gotlib, I. H. (2011). Quality of life and impulsivity in bipolar disorder. Bipolar Disord. 13, 303-309. doi: 10.1111/j.1399-5618.2011. 00919.x

Vilela, J. A. A., Crippa, J. A. S., Del-Ben, C. M., and Loureiro, S. R. (2005). Reliability and validity of a Portuguese version of the young mania rating scale. Braz. J. Med. Biol. Res. 38, 1429-1439. doi: 10.1590/S0100-879X20050009 00019

Watkins, H. B., and Meyer, T. D. (2013). Is there an empirical link between impulsivity and suicidality in bipolar disorders? A review of the current literature and the potential psychological implications of the relationship. Bipolar Disord. 15, 542-558. doi: 10.1111/bdi. 12090

Young, R. C., Biggs, J. T., Ziegler, V. E., and Meyer, D. A. (1978). A rating scale for mania: reliability, validity and sensitivity. Br. J. Psychiatry 133, 429-435. doi: 10.1192/bjp.133.5.429

Conflict of Interest Statement: The authors declare that the research was conducted in the absence of any commercial or financial relationships that could be construed as a potential conflict of interest.

Copyright (c) 2017 Lima, Malloy-Diniz, de Miranda, Da Silva, Neves and Johnson. This is an open-access article distributed under the terms of the Creative Commons Attribution License (CC BY). The use, distribution or reproduction in other forums is permitted, provided the original author(s) or licensor are credited and that the original publication in this journal is cited, in accordance with accepted academic practice. No use, distribution or reproduction is permitted which does not comply with these terms. 